\title{
Real-time Positioning Measurement using Intelligent Shoes
}

\author{
Yahong Li*, Wanyan Guo, Jingzhe Fang and Shujie Sun \\ Shenyang University of Technology Software School, Shenyang, Liaoning, 110023, China \\ ${ }^{*}$ Corresponding author
}

\begin{abstract}
The elderly travel safety has attracted more and more attention, this paper studies a real-time positioning measurement using intelligent shoes system that can locate the position information of the elderly in real time. The project is a positioning system integrated in the bottom of the heel part of the elderly. The positioning system of interaction with the server transmits location information of the elderly through GPS and SIM models in the bottom of the shoes. So the mobile clients achieve tracking and monitoring for the elderly travel through the interaction between the server and the mobile client. This paper includes the system function design, system structure design, in-depth study of the technical route and key problems. In the functional design, it is explained how to perceive the old location information and real-time positioning display, transmit travel data and process electronic fence function. In the structural design includes construction of the elderly travel information acquisition subsystem, data transmission subsystem, data processing and service interface subsystem and real-time positioning, intelligent fence subsystem, forms a complete system development structure.
\end{abstract} fences

Keywords-Intelligent shoes; Real-time positioning; intelligent

\section{INTRODUCTION}

With the arrival of the inevitable ageing of population, it is quite urgent to be lost for the aged. People pay more attention to the whereabouts of the elderly, and now the advanced program is to allow the elderly to carry communication tools to get the whereabouts of the elderly. Frequently adopted approach is to get the position by calling the elderly, but old people often forget to bring a cell phone or even cannot use the current smart phone and to achieve the purpose of monitoring the elderly. Imagining that the elderly cannot remember the phone number or act of their children, the consequences could be disastrous.

It is necessary to wear the shoes for everyone, the positioning system places the bottom as the part of shoes, collecting GPS positioning information obtained through the SIM module through ARM to send to the remote server. The data collected on the remote server is analyzed, which will be transmitted to the PC terminal and Android terminal display.

The project is based on the Android platform, using GPS positioning system to collect the elderly travel information and using the SIM module to send the position information to the server. Being processed by the server, through the mobile Internet, travel information is sent to the mobile phone client after treatment, so as to realize tracking and monitoring of the elderly travel by the mobile client.

\section{SYSTEM ANALYSIS AND DESIGN}

The elderly travel information from the GPS system is sent to the Web server system by the SIM module. The communication between collecting end and the server adopts Socket communication technology to achieve the positioning information stored in the database. The system based on Web server using PHP technology to access the database table positioning information, after the data processing, using JSON format to XML file to the mobile phone terminal.

\section{A. Structure of System}

Through the GPS and the SIM chip module at the bottom of the old shoes the location information of the elderly is achieved and transmitted. The server system process and send the transmission data to the mobile phone by using JSON data and XML file. It is implemented to track and monitor the elderly travel using mobile terminal. So the mobile terminal achieves real-time monitoring for the elderly. Whenever and wherever possible for the old systemarchitecture are shown in Figure 1.

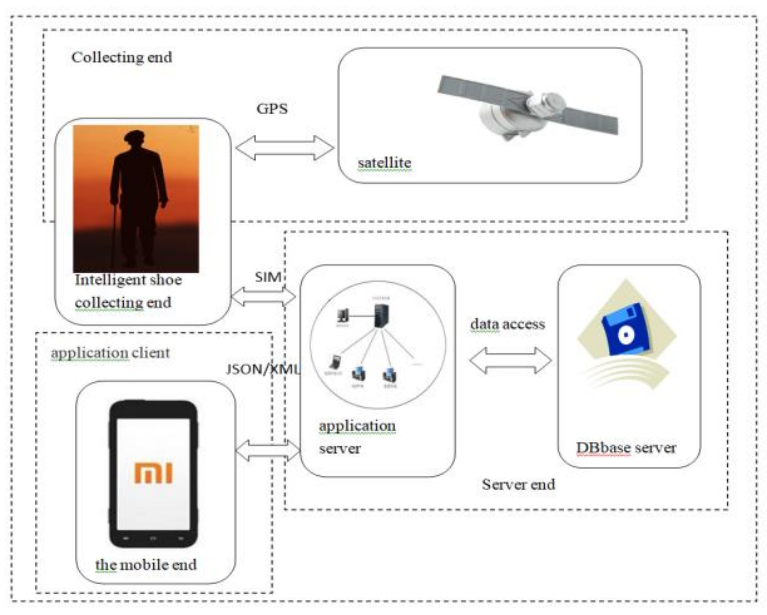

FIGURE I. SYSTEM DIAGRAM

The positioning system consists of a control terminal Arm9, data acquisition and data transmission terminal of GBLOX GPS terminal SIM900. GPS acquires current geographic information through the satellite and the data is sent to the control terminal. When the control terminal receives data, it 
will encode the data and then transmit the data to the data transmission terminal, data transmission terminal sends the data to the remote server by sending data to the remote server network. The collected analys is, the data stored in the database, using Android program for network map when the terminal location information will go to the remote server to obtain the current binding equipment, remote server data will be read out from the database, forwarding to the end of Android.

\section{B. System Function Module}

System function is divided into five modules, the system function structure shown in Figure 2.

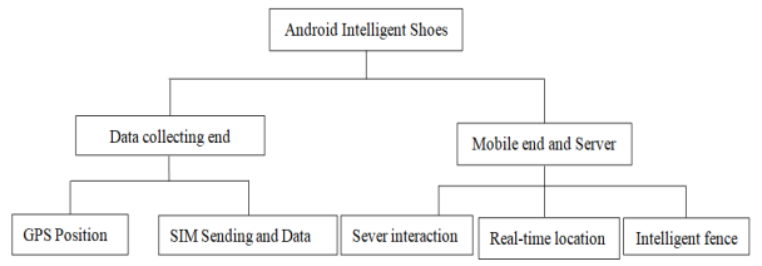

FIGURE II. SYSTEM FUNCTION STRUCT URE

\section{1)GPS positioning}

Position information module in a certain frequency receives automatically the location information by the shoes. The system adopts ARM9 as the control terminal. Power system will first in itialize the hardware and software environ ment, the serial configuration register. The external detection module is working, if everything is normal, the GPS collecting module will start to send commands, positioning information is collected back to the serial port, which will trigger the serial interruption and control end call interruptive service procedure. Location information will be collected and analyzed, using the serial port of MINI2440 development board.

\section{2) Remote transmission of location information}

MINI2440 development board through the serial port communicate with the SIM module to obtain SIM card IEMI by sending AT commands to SIM module, GPS positioning information and IEMI will be sent to the remote server in the form of data packets in accordance with the prescribed format through TCP communication.

\section{3) Server interaction}

The server processes the received data using PHP technology and stores data in MySQL database. The server every time obtain GPS information using acquisition equipment, analytical data is corresponding JSON data in the XML file and create a file of JSON package form including the latitude and longitude position to generate the corresponding documents for the mobile phone client (Android). In the JSON server, the XML file format parsed on the Android, is gotten from the server according to the local latitude and longitude position.

\section{4)Real-time positioning}

In the Android mobile end, the location information is displayed in WebView widget of browser. The real-time position of map is developed on mobile terminal us ing Baidu API. The latitude and longitude of Baidu API as the known information is the use of BMAP for positioning method. Positioning addressing is displayed according to the latitude and longitude of the geographic.

\section{5)Intelligentfence}

To find a planning route on the map, the use of mobile phone navigation is to find the precise position of elderly. The place of the elderly destination is fixed as a circle and takes fixed length as radius. Automatic selection area is defined as the intelligent fence to find the old man, at the same time as the warning area to prevent the elderly from unnecessary injury and property damage.

\section{The System Mainly Solves the Problem}

$\mathrm{N}$ The development of the system is mainly to solve three problems, including the receiving and sending of the GPS signal, the server and the collection terminal, the mobile terminal communication and the mobile terminal display.

\section{1)GPS signal processing}

The location information is acquired and analyzed by sending GPS commands in serial port of MINI2440 development board. The MINI2440 development board through the serial port communicates with the SIM module to obtain IEMI of the SIM card, sending AT commands to SIM module. GPS positioning information and IEMI will be gotten using TCP communication with the SIM module and be encapsulated as data packets in accordance with the prescribed format. Then the data form is sent to the remote server.

2) Communication among the Server, the collection end and the mobile phones

The program is developed in server using PHP technology and information is stored in MySQL database. At regular intervals the server obtaining the position information by GPS acquisition equipment sending, storing the analytical data into the corresponding data table for queried after days. Server encapsulates the information of longitude and latitude into a XML file of JSON package form. After parsing XML or JSON file, the server send data to the mobile phone client (Android). The mobile phone of android terminal parses data of the JSON or the XML file format which be received from the server. The mobile phone accesses the parsed longitude and latitude and displays the position in the WebView of browser.

\section{3)Mobile mobile display processing}

A WebView widget is used in the showing map of the position at the Android webpage. The mobile terminal is developed using the Baidu map API in the Java file. The basic position function is realized using the methods of LocationManager and location and message transmission is realized by the Handler class. To achieve parameters, the realization of the location of map can be used in the function of Lat Lng(),the thread knowledge and the analys is of JSON data. Through the information of the latitude and longitude, the position display of the map is realized by the method of getLocation with Geocoder in BMAP according to antigeocoding of the latitude and longitude. The mobile phone can automatically select the most efficient way to find the destination of the old man. 


\section{DESIGN AND IMPLEMENT ATION OF COMMUNICATION FUNCTION}

GPS positioning information through the SIM module is sent to the server, the server receives the positioning information to process and store into the data table. The PHP server application processes the data by accessing the database table and sends them to mobile phone terminal. So the realization of the communication module is important.

\section{A. Mobile Terminal Design}

The method of requesting service information throught HTTP is commonly used in two ways: GET and POST. The format of the HTTP request consists of four parts : <requestline $>,\langle$ headers $>,\langle$ blankline $>,<$ request-body $>$. In a request of HTTP, there is on the first line (request line) to indicate the type of request, the resources are to be accessed and the HTTP version is to be used. The header is used to explain the specific additional information of server. After a blank line you can add any other data called the body. GET request parameters of a sequence of key/value (query string) as query string appended to the URL whose length is limited by the web browser and web server. POST request is used to transmit form information, so it is necessary that Content-type is the type of application/xwww-form-URL encoded. User fields are transmitted as key/value parameters using POST method on a web form. The use of the Post method is more than that of the GET method in which the Request-Line added directly in URL and submitted through GET, so username and password are clearly shown on the URL, and the data as the part of URL is visible. It is the loss of security. However, the POST method is the data into the body part and not revealed in the address. So it maintains a certain security. Therefore, this paper chooses the Post method for data interchange. The specific solution procedure of Android application is as followed.

1) The DefaultHttpClient class creates an object of class httpClient which extands the class of HttpClient

HttpClient is the realization of HTTP commun ication library of a client not a browser whose goal is to send and receive HTTP messages. The class of HttpClient is saved as a buffer and executed JavaScript code embedded in HTML pages, match the content type, reformat the request / redirect URI., or other not related with transport. The class of HttpClient do not impose to limits the execution of the process request, but in the connection management, the state management, the authentication and the redirected processing there is the specific implementation in detail. These should be easy to use additional functions, such as response to the contents of the cache interface, the interface of HttpClient is the default implementation of DefaultHttpClient.

HttpClient httpClient $=$ new DefaultHttpClient ()$;$

2) Create a HttpPost object extands the class of httpPost, using the HttpPost to send data

By creating the HttpPost object, using the httpPost method, set the parameters of the method for the server URL address.Such as :HttpPost httpPost = new HttpPost(url).

3) Create parameters of a List object with a data type of NameValuePair parammeters.
The List object of Name ValuePair is the name/value node type, in which the corresponding data is saved through using the node of the key/value type.

Lis $\mathrm{t}<$ NameValuePair $>$ params $=$ newArrayLis $\mathrm{t}<$ NameValuePair $>()$;

params .add(new BasicNameValuePair(,));

The BasicNameValuePair (,) has two parameters. They are the type of the encapsulated data pairs, the first parameter is the key name, which needs to use double quotation mark, the second parameter is the value which is to communicate the information and the content. The requesting entity of HttpEntity is an interface, with its UrlEncodedFormEntity implementation class to create the object. The back parameter of a String type is used to encoding, and the aim is to ensure that the server unified encoding format.

$$
\begin{aligned}
& \text { HttpEntity httpEntity = } \\
& \text { new UrlEncodedFormEntity(params, "utf-8"); } \\
& \text { httpPost.setEntity(httpEntity); }
\end{aligned}
$$

4) Call the execute() of the httpClient object to send the request and return an object of HttpResponse

Through the interpretation and the implementation of httpClient, the service application receives and sends data to be dealed with and to be ran by the server using the POST method. The application is to response the data which received through the HttpResponse object.

HttpResponse httpResponse $=$ httpClient.execute $($ httpPost $)$;

5) Call the getEntity () method of HttpResponse to obtain the HttpEntity object

String result $=$ EntityUtils.toString(httpResponse.getEntity ()$)$;

Finally, the result of JSON form can be analyzed. It should be noted that operate from the android4.0 is not allowed as the httpClient directly in the UI thread. It needs to add permissions in the AndroidManifest.xml.

$<$ uses-permis sion android:name=

$$
\text { "android.permis sion.INTERNET" /> }
$$

<uses-permis sion android:name=

"android.permission.ACCESS_NETWORK_STATE" />

\section{B. The PHP programming of server}

The server receives the JSON data through the request, in the PHP, the JSON data from the collection is analyzed through the json_decode () method, then you can operate on the data obtained from the Android client.

According to custom communication character 'JSON' to Android and advance packaged JSON name consistent after treatment of \$json_string. The need to pay attention to a situation, when receiving JSON data, may receive data can not directly display the problem, because there are the browser to do processing and corresponding restriction the situation, in this case, although not shown, but the data can be stored in the 
database when needed and then from the database can be determined directly.

\section{Data Format}

In the data transmission data format uses two type online commonly, JSON or XML. XML is an extensible markup language, JSON is a lightweight data interchange format. Text format of using completely language independent, which is the ideal to exchange data format. XML language has a unified format. It is easy to interact with the other remote systems and it is more conventient to share data. It is small and easy to $\mathrm{read} /$ write and to compress, to parse for the data format of the JSON language, and so on. The main advantages and potentials of JSON is smaller than XML, then it saves network flow to transmit. But the drawback is that it does not look better than the XML directly.

public static String createJsonString

(String key,Object value) \{

JSONObject jsonObject $=$ new JSONObject $($ );

jsonObject.put(key,value);

return jsonObject.toString(); \}

In Analysis of JSON data, it need to make it clear to analysis the JSON Object or JSON Array, then determine which analytical technology. On Android platform generally there have two kinds of analytical techniques to be adopted: JSONObject and Google in the org.json package in GSON library of Android open source. In addition, some of the third open source libraries such as Jackson and FastJSON is also very good.

JSONObject \{"key": "value" \},

JSONArray [\{"key": "value" \}, \{"key": value" \}]

JSONArray contains more than one JSONObject. Through the JSONObject to access, jsonObject.getXXX ("key") to get the corresponding data. Create JSONObject to receive a JSON data, and then create an array of JSONArray objects to obtain an array of JSONObject in the JSONArray, each of array is assigned to a JSONObject in the cycle structure which is obtained by using JSONObject.getXXX () method.

\section{Focus and Difficulty During Design}

\section{1) ARM interrupt mode switching}

Because the serial port's speed is very slow, CPU is blocking when it is waitting for serial port's data. In order to making CPU do other things while waiting for serial data, the interrupt is used. Serial data is received by using the interrupt procedures.

2) Socket communication with the collection client and the server

The collection client sends the acquired data to the server through Socket communication.

3) JSON file transfer and analysis

Mobile phone and server exchange information through the JSON.
4) AJAX asynchronous transfer

a) Set URL into XMLHttpRequest by creating Object of XMLHttpRequest.

b) Set a callback function to XMLHttpRequest.

c) Asynchronously send the request of XMLHttpRequest.

d) Display the data by server responsing and calling the callback function.

5) Data interaction between PHP and database:

Through mysql_connect () and mysql_select_db () connectivity is realized between the PHP and database. The operation of sqlite CRUD is achieved through function mysql_query (\$sql).

\section{CONCLUSION}

With the arrival of an aging society, the safe travel of the elderly also will usher in an important period. This paper puts forward the construction scheme of multifunctional shoes elderly intelligent system based on Android, the discussion and in-depth analysis at the same time, the technical route and key problems of the program design combined with the existing problems of the elderly travelers. Through the realization of positioning systems embedded into the old shoes, the elderly can be monitored everytime and everywhere for solving the elderly travel safety and monitoring role to a certain extent. It brings positive social value for the development of the society and more help to improve the elderly travel safety.

\section{REFERENCES}

[1] Hieu. Loosely coupled GPS/INS integration with Kalman filtering for land vehicle applications[J].Control, Automation and Information Sciences (ICCAIS), 2012 International Conference on,2012.

[2] Esteban.EURAMET Project 1156, GPSCALEU:Results of two years GPS receiver calibration campaign[J].2012

[3] Luo Junzhou, Wu Wenjia, Yang Ming. Mobile Internet: Terminal Net work and Service [J] .Journal of Computers, 2011,11.

[4] HEWeiwei, WANGAihua, MAYue. Study on GPS vehicle terminal communication technology based on GPRS [J]. Journal of Computer Applications.2008,11.

[5] ZhangLu, ChenChunlei. Design of SMS Control System Based on GSM[J]. China Science and Technology Information ,2006,3

[6] HuKegang, WangShuxun, LiuLihong.Wireless Location Technology in Mobile Communications[J]. Journal of Jilin University (Information Science Edition).2005,4

[7] BiJun, GuanWei, ShenJinsheng.Design and Implementation of GPS / GSM Hand-held Locator. Volume 19, No. 3, June: 3 2005, 8-41

[8] CAIChengsen. Study on Key Technology of Mobile Target Real-time Monitoring System Based on GPRS Network [D]. Shandong University .2005. 